\title{
Is Systems Complexity a Property in a Functional State Space of Each System?
}

Andy E. Williams, Nobeah Foundation, Nairobi, Kenya

awilliams@nobeahfoundation.org

\begin{abstract}
:
Human-Centric Functional Modeling has been proposed as a universal approach towards defining representations of the behavior of systems in terms of "functional state spaces". Such descriptions are hypothesized to be complete in terms of having the capacity to represent all the behavior the system is capable of displaying. This paper provides a brief exploration of Human-Centric Functional Modeling, how it might be used to model the behavior of the cognitive system in terms of a functional state space, and how cognitive complexity might be defined as a property in this functional state space of the cognitive system. Assuming that functional state spaces can be defined for other systems as well, this paper explores the questions that must be asked in considering whether this hypothetical expression for complexity in the functional state space of the cognitive system systems represents a general pattern for defining complexity in any functional state space, and whether complexity is therefore a well-defined property of the functional state space of any system.
\end{abstract}

\section{Introduction}

Human-Centric Functional Modeling or HCFM [1], [3] describes systems as having a set of functions through which the system might transition from one state to another, where each state is defined in terms of such functions. These states described in terms of functions are referred to as "functional states", each of which might be composed of other functional states. All of the functions of a system within a given domain of behavior map between functional states belonging to the same category. Any given category of functional states then forms a "space" of functional states or a "functional state space".

As an example, the cognitive system can be represented as a space of concepts connected by reasoning processes which act as functions that enable the cognitive system to transition from one concept to another. This representation of cognition as moving through a "conceptual space" has been used to define a model of artificial cognition (Artificial General Intelligence or AGI [2]).

In network terminology this functional state space is represented by a graph containing a network of nodes each representing a functional state, where those functional states are connected by edges representing the processes through which one functional state might transition to another. Where an ontology defines a closed set of concepts and a finite set of reasoning processes that connect each concept, HCFM utilizes this open network to facilitate the definition of every possible concept and every possible reasoning process connecting each concept. If the graph of the functional state space of the cognitive system (the conceptual space) represents the complete human meaning of each concept and each reasoning process, it is therefore a complete semantic model. If this functional state space represents the complete set of behaviors our thoughts are capable of, even if we don't know how our minds work (that is even if we don't know how our minds are able to "think"), then it is capable of representing all thought.

The current understanding generally accepted in cognitive psychology is that human thought consists of two modes of thinking, namely type 1 (fast or intuitive) reasoning, and type 2 (rational methodical or slow) reasoning. Both of these modes are represented as paths in the conceptual space defined by Human-Centric Functional Modeling, with type 1 reasoning potentially being represented by a direct path between concepts, and with type 2 reasoning being represented by an indirect path that is 
expressed in terms of smaller paths tjat connect intermediate concepts. Type 1 and type 2 reasoning in conceptual space potentially represent a more general pattern in all functional state space. Representing problems in functional state space as the gap between two regions in that space, and representing solutions as the processes providing that path, then there are two types of solutions to every problem in every system. One type of path is direct, which we might call the "uncomputable" solution. In our cognitive system this corresponds to type 1 reasoning which cannot be computed. Another type of path is in terms of smaller existing path segments, which we might call the "computable" solution. In our cognitive system this represents type 2 reasoning.

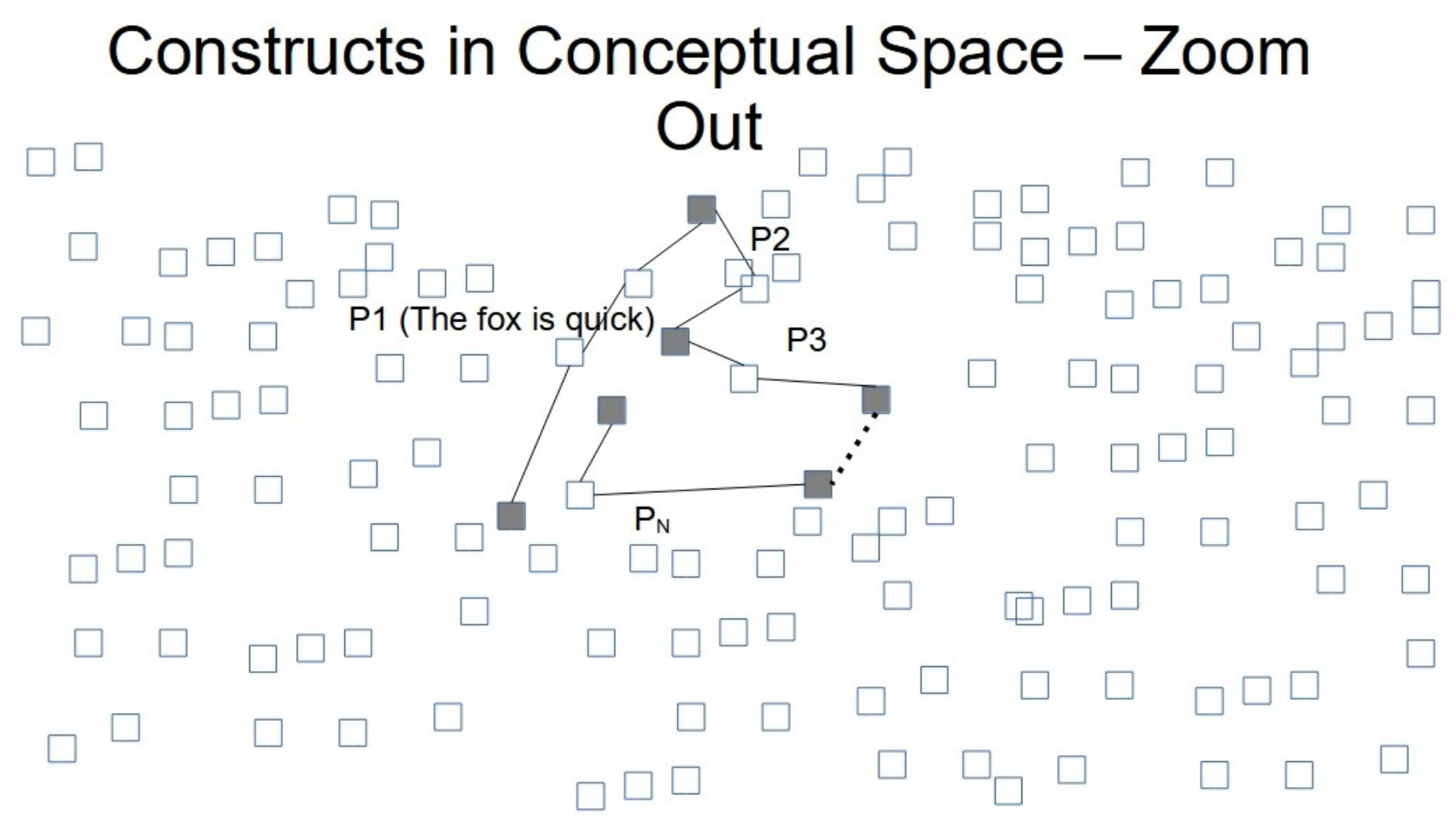

Figure 1: In conceptual space, zooming out to the high level picture, a concept is a node (represented by each square box) and reasoning is a path (represented by a line). 


\section{Constructs in Conceptual Space - Zoom In}

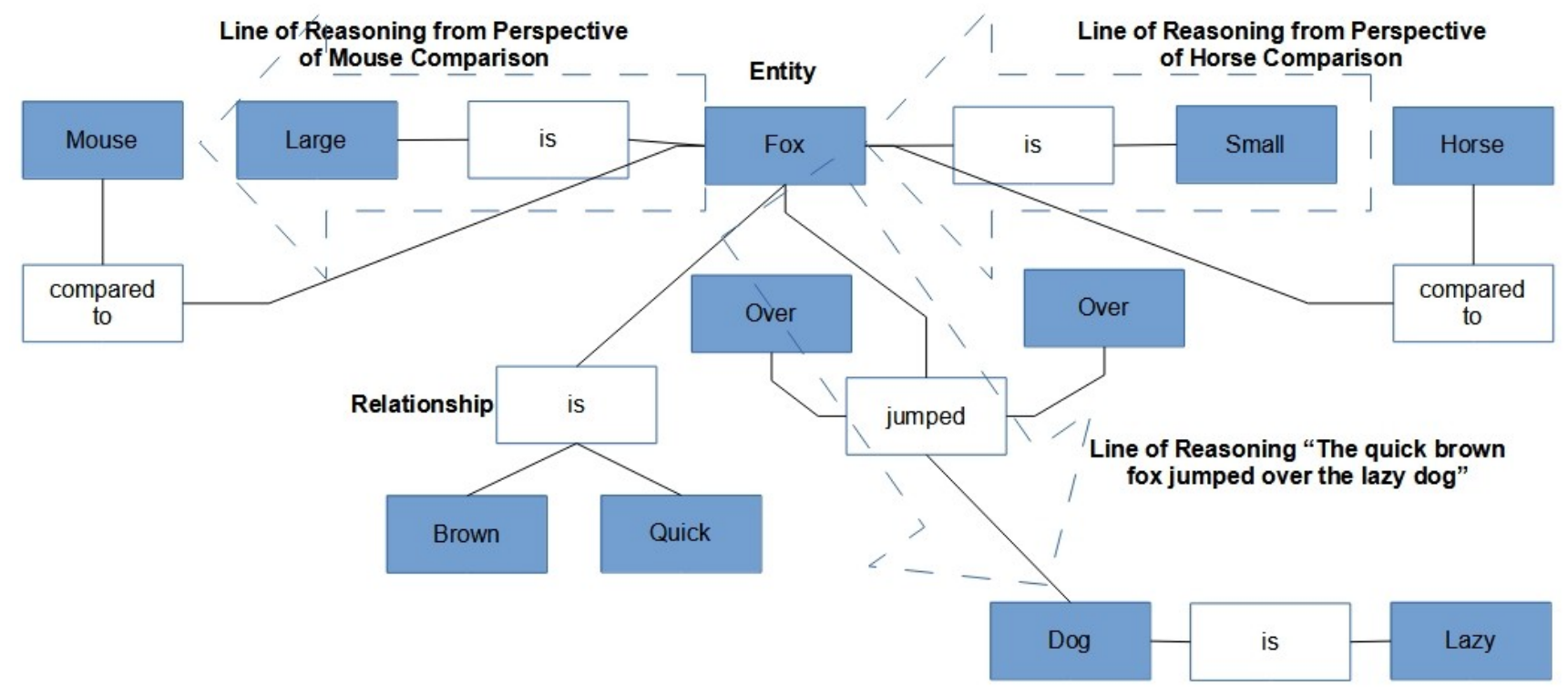

Figure 2: In conceptual space it is possible to zoom in to view specific concepts and the reasoning relationships between them as well. These relationships might be viewed from different perspectives. As an example, from the perspective of the horse its relationship with the fox in terms of size is that the fox is small.

In addition to reasoning type, every property of the cognitive system (including "complexity", and including general problem-solving ability or "intelligence") is potentially defined by a mathematical expression in the functional state space of the cognitive system [1]. Because these expressions are based on our understanding of human cognition they are human-centric. If every other system (e.g. systems in physics [8], biology [9], mathematics [10], software [11], etc.) can potentially be understood in terms of a functional state space as well, then such functional state spaces might also define a complete semantic representation (a complete representation of human meaning) for those other systems too. This enables us to potentially understand every other system using the same approach, which is relevant to problem solving in every discipline from physics, mathematics, biology, and psychology to sustainable development.

\section{Hypothesis of Complexity in Conceptual Space}

Qualitatively we expect that concepts linked through shorter reasoning processes will be closer in conceptual space than concepts linked by longer reasoning processes. We also expect that more general concepts occupy a larger region in conceptual space, and more specific concepts occupy a smaller region in conceptual space. A generalization might then contain many concepts, represented by a larger region in conceptual space that encloses the smaller regions representing those concepts. A

specification of a concept would be expected to contain fewer concepts, represented by a smaller region in conceptual space. We would expect that the more a concept is specified the greater the amount of reasoning that links the concept to other concepts and the smaller the region in conceptual space that represents the concept (that is, the more precisely it is defined). Assuming that a problem is represented in conceptual space as the lack of a path from one concept to another, and assuming that a solution is 
represented in conceptual space as the path which provides that reasoning, then it is hypothesized here that the complexity of a solution is the distance in conceptual space multiplied by the linear density of concepts along that solution path. The complexity of a problem is hypothesized here to be the complexity of the least complex solution. The complexity of a single concept is hypothesized here to be represented by the product of its volume and its volume density in conceptual space.
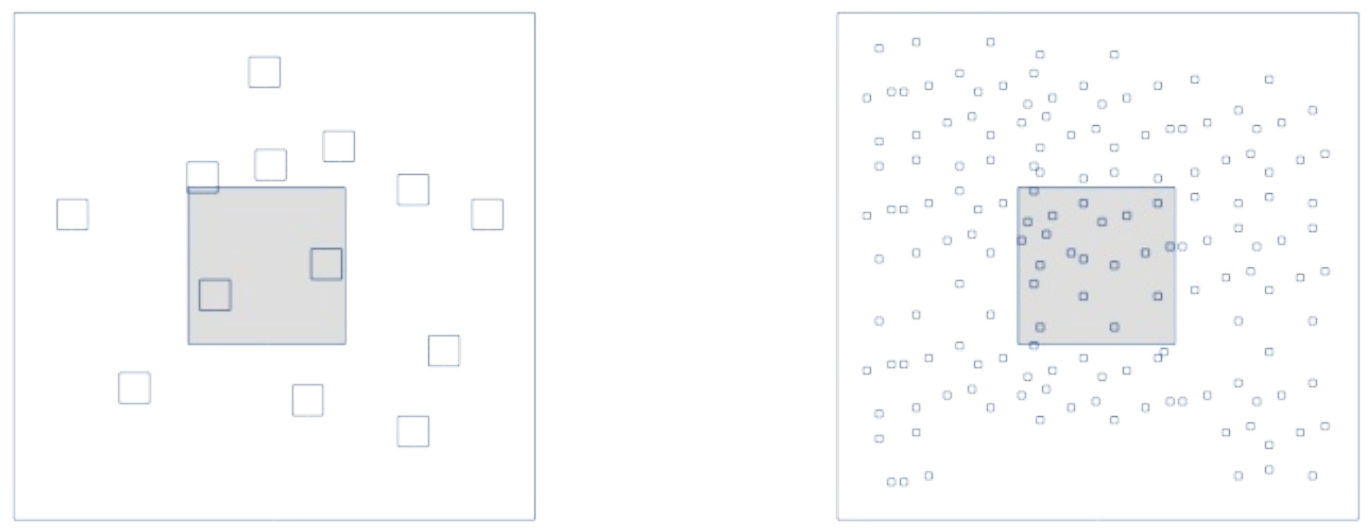

Figure 3: In conceptual space, a concept (gray square) is less complex when the density (resolution) of concepts is lower (left) and more complex when it is higher (right).

We can qualitatively verify these statements as in table 1.

\begin{tabular}{|c|c|c|c|}
\hline Concept 1 & Concept 2 & $\begin{array}{l}\text { Example of Reasoning } \\
\text { Relationship }\end{array}$ & $\begin{array}{l}\text { Complexity of Reasoning } \\
\text { Relationship }\end{array}$ \\
\hline Cat & Mouse & Cats eat mice. & $\mathrm{C}_{1}$ \\
\hline Cat & Paint & $\begin{array}{l}\text { Cats might step into a bucket } \\
\text { of paint. }\end{array}$ & $\mathrm{C}_{2}>\mathrm{C}_{1}$ \\
\hline $\begin{array}{l}\text { Riemann- } \\
\text { Lebesgue } \\
\text { lemma }\end{array}$ & Fourier transform & $\begin{array}{l}\text { A lemma is a subsidiary or } \\
\text { intermediate theorem in an } \\
\text { argument or proof. The } \\
\text { Riemann-Lebesgue lemma in } \\
\text { mathematics states that the } \\
\text { Fourier transform or Laplace } \\
\text { transform of an } \mathrm{L}^{1} \text { function } \\
\text { vanishes at infinity. }\end{array}$ & $\mathrm{C}_{3}>\mathrm{C}_{2}$ \\
\hline
\end{tabular}

Table 1: Concepts and the complexity of the relationships between them, where the length of the reasoning is taken as a simple proxy for distance in conceptual space.

It is hypothesized here that the required density of concepts in any reasoning connecting two concepts is determined by the density (complexity) of the more complex of the two concepts. In other words a problem defined in terms of reasoning from one extremely specific concept to one very general concept must address the complexity of the extremely specific concept.

\section{Validating the Hypothesis of Complexity in Conceptual Space}

Though it is still very powerful in its current form, the idea of conceptual space is incomplete. There are a number of problems that must be solved before any implementation of a conceptual space can be used to semantically store concepts and reasoning. One is that distances are not precisely quantified. 
Another is that there is not yet a representation of concepts and reasoning relationships that is fully self-contained (independent of any lookup table). However, because any other functional state space might also be represented by a graph containing a network of nodes representing functional states connected by edges representing the processes through which the system transitions between states, then solving these problems for any functional state space solves these problems for every functional state space, including the conceptual space.

Assuming that functional state spaces can be defined in other disciplines such as physics [8], biology [9], mathematics [10], computing [11], or sustainable development [12], there is a strong value proposition for physicists to solve these problems for the functional state space representing the physical universe, in having mathematicians solve these problems for the functional state space representing mathematical relationships, and in having other technical experts solve these problems for the functional state spaces representing the systems relevant to their own disciplines. This value proposition is the opportunity to vastly increase our collective capacity to navigate complexity and to solve problems in physics and mathematics as well as in any other disciplines that functional state spaces might be defined for. Once data regarding physical systems can be represented semantically for example, then all components of all theories can be tested against all data, thereby removing bottlenecks to science such as the need to discard some theories as "fringe" in order to limit the focus of precious scientific attention. Once mathematical relationships can be defined in terms of some functional state space, perhaps as a geometry in that space, then every relationship is potentially related to every other by some geometric transformation (translation, rotation, reflection). New relationships might then be discovered through such simple geometric transformations.

Determining the ways in which the representation of functional state space is incomplete are some of the questions that must be asked in answering the question of whether systems complexity is a property of the functional state space of the system given by the expression: Complexity $=$ linear density of functional states $*$ distance in functional state space.

\section{Functional State Spaces of Systems Related to Other Disciplines}

This paper has explored how our minds as cognitive systems can potentially be represented as moving through a space of thought, that is, a conceptual space. This space of thought is a functional state space since each point in it represents a concept and since each concept is a functional state in that it is defined by the reasoning processes that function to transition the mind from that concept to other concepts. If our cognitive systems can be represented as moving through a space of thought, then it is possible to represent all other systems as moving through their own subset of this thought space. This subset of thought space is a functional state space that the system we are thinking about moves through as our thoughts about it change. We don't need to make any assumptions that this subspace of our "thought space" is contiguous for this to be true. Alternatively, completely independently of us, any system can be represented as moving through its own functional state space as it changes, regardless of whether or not we think about it. All systems then move through such a functional state space, either in our minds or in the real world. If everything we can conceive of in our thoughts falls within a functional state space, and if we can conceive of the entire physical universe, then it is possible to represent the entire physical universe as moving through its own functional state space as well.

There is some existing work suggesting the possibility of defining a functional state space through Human-Centric Functional Modeling to represent the physical universe, since doing so might be equivalent to the use of constructor theory [7], a class of theory which has been described as seeking to "express all fundamental scientific theories in terms of a dichotomy between possible and impossible 
physical transformations". There is also some existing work suggesting the possibility of defining functional state spaces representing other biological systems such as consciousness [4], [5], [6].

Assume that this functional state space does allows us to create this mathematical definition for complexity of thought where that definition can be expressed in terms of this conceptual space as the functional state space of the cognitive system. Since every other system potentially also moves through a functional state space, then if complexity as a property belongs to functional state space rather than to any particular system, the same expression might be used to understand the complexity in all other systems as well. If complexity is represented by the distance in conceptual space between the concepts that must be bridged by any solution, multiplied by the linear density of concepts along that reasoning path, then complexity of a physical, biological, computing, or other system might be represented by the exact same expression in the functional state space of the system. If so, this shows the importance of having a universal way of understanding all systems. Namely it enables our understanding of any one system to potentially be reused in understanding any other.

\section{Conclusions}

That the complexity of a transition by any system might be essentially given by the product of number of functional states the system must transition through and the distance between those states seems plausible but remains to be proved. Proving it will require elaborating the representation of functional state spaces to better define properties like distance. If functional state spaces can be used to exponentially increase our collective capacity to navigate complexity and solve problems for each system as predicted, and if this exponential increase in general problem-solving ability is not reliably achievable otherwise as predicted as well, then proving the validity of this representation of complexity is one of the most important challenges today. It is then one that is deserving of significant engagement.

One potential path towards engaging a significant effort in the target disciplines of mathematics, physics, biology, computer science, and other fields to prove that complexity is a property in a functional state space and to determine what that solution is, is to organize a grand challenge. Before such a grand challenge can be organized it's important to determine the questions that must be answered by the winning solution. The questions which the analysis of this paper indicates are most important are how systems relevant to each discipline can be represented in terms of one or more functional state spaces, and how the missing elements in representing functional state space can be addressed.

\section{References}

[1] Andy E. Williams, A Revolution in Systems Thinking?, Proceedings 2021 Congress of the World Organization of Systems and Cybernetics (WOSC), Accepted

[2] Williams A.E. (2020) A Model for Artificial General Intelligence. In: Goertzel B., Panov A., Potapov A., Yampolskiy R. (eds) Artificial General Intelligence. AGI 2020. Lecture Notes in Computer Science, vol 12177. Springer, Cham. https://doi.org/10.1007/978-3-030-52152-3 38

[3] Williams, Andy E. "Human-Centric Functional Modeling and the Unification of Systems Thinking Approaches: A Short Communication." Journal of Systems Thinking (2021): 5-5.

[4] Signorelli, Camilo Miguel, Quanlong Wang, and Bob Coecke. "Reasoning about conscious experience with axiomatic and graphical mathematics." Consciousness and Cognition 95 (2021): 103168.

[5] Signorelli, Camilo Miguel, and Daniel Meling. "Towards new concepts for a biological neuroscience of consciousness." Cognitive Neurodynamics (2021): 1-22.

[6] Signorelli, Camilo Miguel, Joanna Szczotka, and Robert Prentner. "Explanatory profiles of models of consciousness-towards a systematic classification." (2021). 
[7] Deutsch, D. Constructor theory. Synthese 190, 4331-4359 (2013). https://doi.org/10.1007/s11229$\underline{013-0279-\mathrm{z}}$

[8] Williams, A. E. (n.d.). The Importance of Representing the Physical Universe or Virtual Universes in Functional State Space to the Progress of Physics. Retrieved from osf.io/preprints/africarxiv/54qrz [9] Williams, A. E. (n.d.). Modeling Biological Systems Using Functional State Spaces. Retrieved from osf.io/preprints/africarxiv/6k4z5

[10] Williams, A. E. (n.d.). Are Mathematical Relationships Represented by Geometries in Some Functional State Space?. Retrieved from osf.io/preprints/africarxiv/mytwe

[11] Cognitive Computing and its Relationship to Computing Methods and Advanced Computing from a Human-Centric Functional Modeling Perspective", In: Pal R. and Shukla K. P. (eds), SCRS

Conference Proceedings on Intelligent Systems, SCRS, India, 2021, pp. 107-122.

https://doi.org/10.52458/978-93-91842-08-6-9

[12] Williams, A. E. (n.d.). Is Sustainability a Pattern of Solution in a Functional State Space?.

Retrieved from osf.io/preprints/africarxiv/cb2xh 